\title{
Waye Induced Pressures on Submerged Plates
}

\author{
By William W. Durgin ${ }^{1}$ and Jong C. Shiau ${ }^{2}$
}

\section{INTRODUCTON}

The prediction of forces, or more precisely, the pressure distributions, experienced by submerged structures due to the passage of gravity waves has become important with the advent of large offshore structures. If the size of a structure is small compared to the wavelength then the forces can be evaluated by the assignment of suitable drag and inertia coefficients using an approach similar to that of Morison (6) in which case it is necessary to determine the coefficients experimentally for any given geometry (2). If the size of the structure is a significant fraction of the wavelength, the preceding method is invalid and a more complicated analysis such as the diffraction theory of Garrison and Rao (1) is needed. Unfortunately, the application of diffraction theory for a given geometry is exceedingly difficult unless one assumes that the structure size is small compared to the wavelength, which may not be realistic.

In order to circumvent the evaluation problems of diffraction theory an approximate analysis based on thin airfoil theory was developed. The analysis to be presented is developed for a thin horizontal flat plate, although extension to other objects is not difficult. One applicable physical situation is that of a protective cap over a vertical water intake. Typically such a cap might be $50 \mathrm{ft}(15 \mathrm{~m})$ wide submerged approximately mid-depth in water $25 \mathrm{ft}(8 \mathrm{~m})$ deep. Also typically, wavelengths of the order of $200 \mathrm{ft}(61 \mathrm{~m})$ would be experienced making the plate width of the order of one-fourth the wavelength. The question of wave induced loadings on such structures associated with nuclear power stations prompted the investigation presented herein.

\footnotetext{
'Asst. Prof. of Mech. Engrg, and Sr. Research Engr., Alden Research Labs., Worcester Polytechnic Inst., Worcester, Mass.

${ }^{2}$ Research Engr., Alden Research Labs., Worcester Polytechnic Inst., Worcester, Mass.
} 
In the analysis, the plate is considered as a thin airfoil in an oscillating flow and as such is replaced by a vortex sheet. It is assumed that since the frequency of oscillation is relatively high no vortices are shed from the plate [this condition is similar to the small wave amplitude to structure size ratio of Garrison and Rao (1)]. Further, the free surface and bottom boundary conditions are approximately satisfied in the sense that the Froude number, based on frequency and submergence, is taken as small and only a few images of the sheet in surface and bottom are considered. Robertson (7) presents the small Froude number approximation and points out that it implies a rigid surface which, in this case, means the original waveform can again be found some distance downstream from the plate.

The evaluation of the strength distribution of the vortex sheet is carried out for the case of sinusoidal waves wherein the resulting integral equations are solved numerically by replacing them with two sets of $N$ simultaneous linear equations ( $N=10$ is sufficient and can be performed easily with a small computer). Finally the differential pressure distribution is found using Euler's equation. In the computer scheme used for the present evaluation, only the wavelength, depth, and submergence all normalized with respect to plate width are needed, and the results are given as pressure coefficient distributions for various wave positions over the plate.

In order to test the accuracy, measurements of the pressure distribution due to the passage of waves over a flat plate were made using a wave flume facility. The flat plate, instrumented with pressure sensors, was rigidly supported beneath the water surface and subjected to waves of various amplitude and wavelength. Similar experiments were carried out on a proposed octagonal plate and the measurements compared to those predicted theoretically using the two-dimensional theory.

A force coefficient is calculated to facilitate maximum force calculation and to compare with typical values of the hydrodynamic mass.

\section{THEORETICAL Formulation}

The flow field is considered as potential and two-dimensional. A gravity wave, represented by the velocity potential, $\phi_{w}$, moves to the right with speed $c$ and amplitude $a$ in fluid of mean depth $d$ as shown in Fig. 1. The wave encounters a submerged horizontal plate of width $l$ located a distance, $s$, below the mean

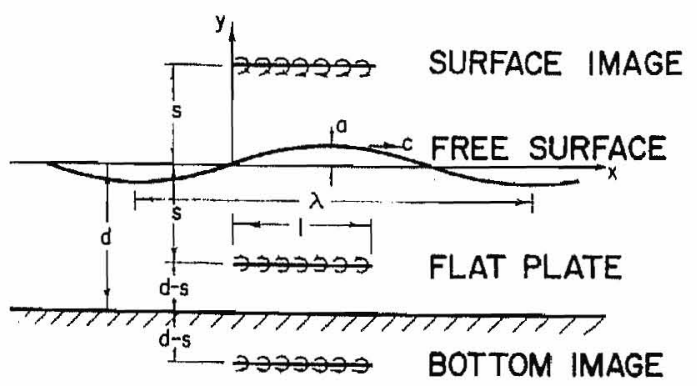

FIG. 1,-Definition Sketch 
fluid surface which can be represented by a perturbation potential, $\phi_{i}$. The central problem is to find $\phi_{i}$ such that Laplace's equation, the free-surface condition, and the bottom boundary condition are satisfied simultaneously.

To effect a solution, the flat plate is replaced by a vortex sheet (3) of strength $\gamma(x, t)$ so that the potential due to the plate alone is

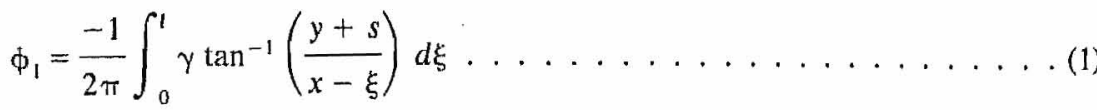

in which $\xi$ is a dummy integration variable.

The no-penetration condition at the bottom requires an image vortex system located a distance, $d-s$, below the bottom given by the potential

$\phi_{2}=\frac{1}{2 \pi} \int_{0}^{1} \gamma \tan ^{-1}\left(\frac{y+2 d-s}{x-\xi}\right) d \xi \ldots \ldots \ldots \ldots$

Representing the free-surface by the linearized condition

$\frac{\partial \phi_{i}}{\partial y}+\frac{1}{g} \frac{\partial^{2} \phi_{i}}{\partial t^{2}}=0 \ldots \ldots \ldots \ldots \ldots \ldots \ldots \ldots \ldots \ldots \ldots \ldots \ldots \ldots \ldots \ldots \ldots \ldots \ldots . \ldots \ldots \ldots$

we will consider only sinusoidal waves of circular frequency $\sigma$ and situations where the Froude number, $F=\sigma \sqrt{s / g}$, is small, therefore, Eq. 3 can be approximated as $\partial \phi_{i} / \partial y=0$. Under these conditions an image system a distance, $s$, above the free-surface is required (7) and the potential is

$\phi_{3}=\frac{1}{2 \pi} \int_{0}^{1} \gamma \tan ^{-1}\left(\frac{y-s}{x-\xi}\right) d \xi \ldots \ldots \ldots$

The bottom image now reflects through the surface to form another surface image while the surface image reflects through the bottom to form another bottom image. The reflections repeat indefinitely but, for the sake of brevity, all those not already listed will be represented by $\Sigma$. Thus, the perturbation potential can be written

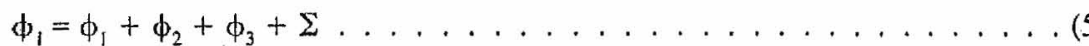

Two conditions are necessary to fix the strength distribution of the vortex sheet. First, that there be no velocity in the $y$ direction at the plate, i.e.

$v=v_{w}+v_{i}=0 ; y=-s ; 0 \leq x \leq l \ldots \ldots \ldots$ (6)

in which $v_{i}=-\partial \phi_{i} / \partial y$; and $v_{w}=-\partial \phi_{w} / \partial y$. The second condition is that the total circulation about the plate be stipulated. The latter condition is usually taken to be the Kutta condition of forcing the trailing edge to be a stagnation point. In this case, we express the circulation condition as no starting vortices shed from the plate. Mathematically this requirement can be expressed as

$\int_{0}^{1} y d x=0 \ldots \ldots \ldots \ldots \ldots \ldots \ldots \ldots \ldots \ldots \ldots \ldots \ldots \ldots \ldots \ldots \ldots \ldots \ldots \ldots \ldots$

On substitution of appropriate expressions, Eqs. 6 and 7 form a pair of integral equations that can be solved giving the strength distribution of the vortex sheet, $\gamma$. 
The horizontal velocities at the plate are then given by (5)

$u^{ \pm}=u_{w}+u_{i}^{ \pm} \ldots \ldots \ldots \ldots \ldots \ldots(8)$

in which $u_{i}^{ \pm}=\mp \frac{\gamma}{2} \ldots \ldots \ldots \ldots$. . . . . . . . . . . .

and the positive superscript refers to the upper surface and the negative to the lower. Finally, the pressure can be found by direct substitution into Euler's equation evaluated at the plate

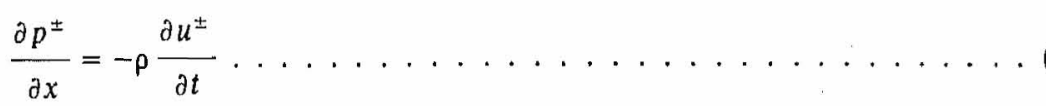

in which the convective terms have been dropped consistent with small amplitude wave theory. Defining $\Delta p=p^{-}-p^{+}$and substituting Eq. 9 into Eq. 10

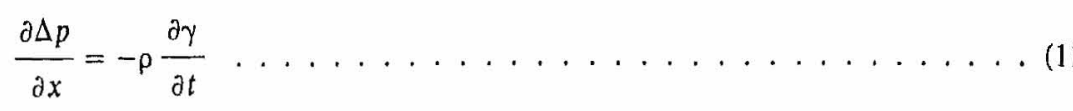

Since there are no shed vortices, the gradient may be integrated around the leading edge of the plate yielding

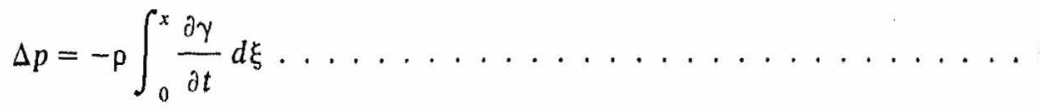

as the induced differential pressure on the plate.

If the oncoming waves are sinusoidal, the potential function may be written 7)

$\phi_{w}=\frac{a g}{\sigma} \frac{\cosh k(d+y)}{\cosh k d} \cos (k x-\sigma t)$

in which $k=2 \pi / \lambda$; and $\sigma=k c$. The vertical velocity due to the wave at $y=-s$ is

$v_{w}=-\frac{\partial \phi_{w}}{\partial y}=-\frac{a g}{c} \frac{\sinh k(d-s)}{\cosh k d} \cos (k x-\sigma t) \ldots \ldots \ldots$

Upon substitution of the appropriate expressions for $v_{w}$ and $v_{i}$, Eq. 6 becomes

$-\frac{a g}{c} \frac{\sinh k(d-s)}{\cosh k d} \cos (k x-\sigma t)-\frac{1}{2 \pi} \int_{0}^{t} \gamma(\xi, t) G(\xi, x) d \xi=0 \ldots$.

in which

$G(\xi, x)=\frac{1}{x-\xi}-\frac{x-\xi}{(x-\xi)^{2}+4(d-s)^{2}}-\frac{x-\xi}{(x-\xi)^{2}+4 s^{2}}+$ other images

Rearranging $\cos \left(k^{\prime} x^{\prime}-t^{\prime}\right)=\int_{0}^{1} \frac{\gamma\left(\xi^{\prime}, t^{\prime}\right)}{U} G\left(\xi^{\prime}, x^{\prime}\right) d \xi^{\prime}$

in which $U=-\frac{2 \pi a g}{c} \frac{\sinh k(d-s)}{\cosh k d} ; \quad k^{\prime}=\frac{2 \pi}{\lambda^{\prime}} ; \quad t^{\prime}=\sigma t$ 
and other primes denote nondimensionalization with respect to $l$, e.g., $x^{\prime}=$ $x / l$. Eq. 7 becomes

$\int_{0}^{1} \gamma\left(x^{\prime}, t^{\prime}\right) d x^{\prime}=0 \ldots \ldots \ldots \ldots \ldots$

Eqs: 17 and 18 which define the strength distribution, $\gamma$, of the vortex sheet may be simplified by noting that $\gamma$ is separable in the form

$\frac{\gamma\left(x^{\prime}, t^{\prime}\right)}{U}=A\left(x^{\prime}\right) \sin t^{\prime}+B\left(x^{\prime}\right) \cos t^{\prime} \ldots \ldots \ldots \ldots$

from which $\int_{0}^{1} A\left(\xi^{\prime}\right) G\left(\xi^{\prime}, x^{\prime}\right) d \xi^{\prime}-\sin k^{\prime} x^{\prime}=0 \ldots \ldots \ldots$

$\int_{0}^{1} B\left(\xi^{\prime}\right) G\left(\xi^{\prime}, x^{\prime}\right) d \xi^{\prime}-\cos k^{\prime} x^{\prime}=0 \ldots \ldots \ldots \ldots \ldots$

$\int_{0}^{1} A\left(\xi^{\prime}\right) d \xi^{\prime}=0 \ldots \ldots \ldots \ldots \ldots \ldots$

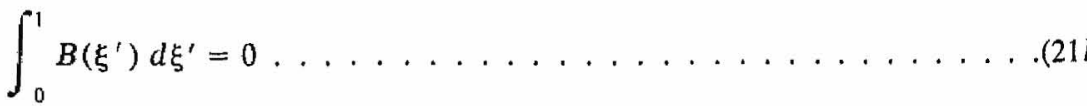

Eqs. 20 and 21 are now in a form suitable for determination of $A$ and $B$. Once $A$ and $B$ are found the pressure is found from Eq. 12 which gives, after some rearrangement

$\frac{\Delta P}{\rho g a}=-\frac{(2 \pi)^{2}}{\lambda^{\prime}} \frac{\sinh 2 \pi\left(d^{\prime}-s^{\prime}\right) / \lambda^{\prime}}{\cosh 2 \pi d^{\prime} / \lambda^{\prime}} \int_{0}^{x^{\prime}}\left[A\left(\xi^{\prime}\right) \cos t^{\prime}-B\left(\xi^{\prime}\right) \sin t\right] d \xi^{\prime}(22$

\section{Experimental. Studies}

Experiments were conducted using a water-wave flume $40 \mathrm{ft}(12 \mathrm{~m})$ long by $4 \mathrm{ft}(1.2 \mathrm{~m})$ wide in which waves were generated by a piston type wave maker executing sinusoidal motion of adjustable frequency and amplitude. A wave absorber, composed of metal lathe turnings, occupied the opposite end of the flume minimizing reflection of the incident wave train.

The horizontal flat plate, shown in Fig. 1, was $1.1 \mathrm{ft}(0.34 \mathrm{~m})$ wide, spanned the width of the flume, and was supported on a number of $0.25-\mathrm{in}$. (6.35-mm) round columns. The 0.375 -in. $(9.53-\mathrm{mm})$ thick plate was constructed of plastic with the central portion hollow to facilitate installation of pressure transducers as can be seen in Figs. 2 and 3. The transducers, three on the upper surface and three on the bottom surface, were of the pressure sensitive transistor type. They were connected to appropriate biasing, balancing, and amplification circuits necessary for adjustment and to provide drive to the galvanometers of the 
recording oscillograph. Calibration was effected by pressurization and evacuation of the hollow central portion of the plate. The wave amplitude was measured with a calibrated parallel wire resistance type wave probe also connected to the oscillograph.

The experimental data were retrieved from the recorder chart by dividing the record between two wave crests into eight equal parts beginning when the crest was over the leading edge of the plate. The pressure at a point on the upper surface was subtracted from the pressure on the corresponding point on the lower surface thus giving $\Delta P$ for comparison with the theory. In plotting

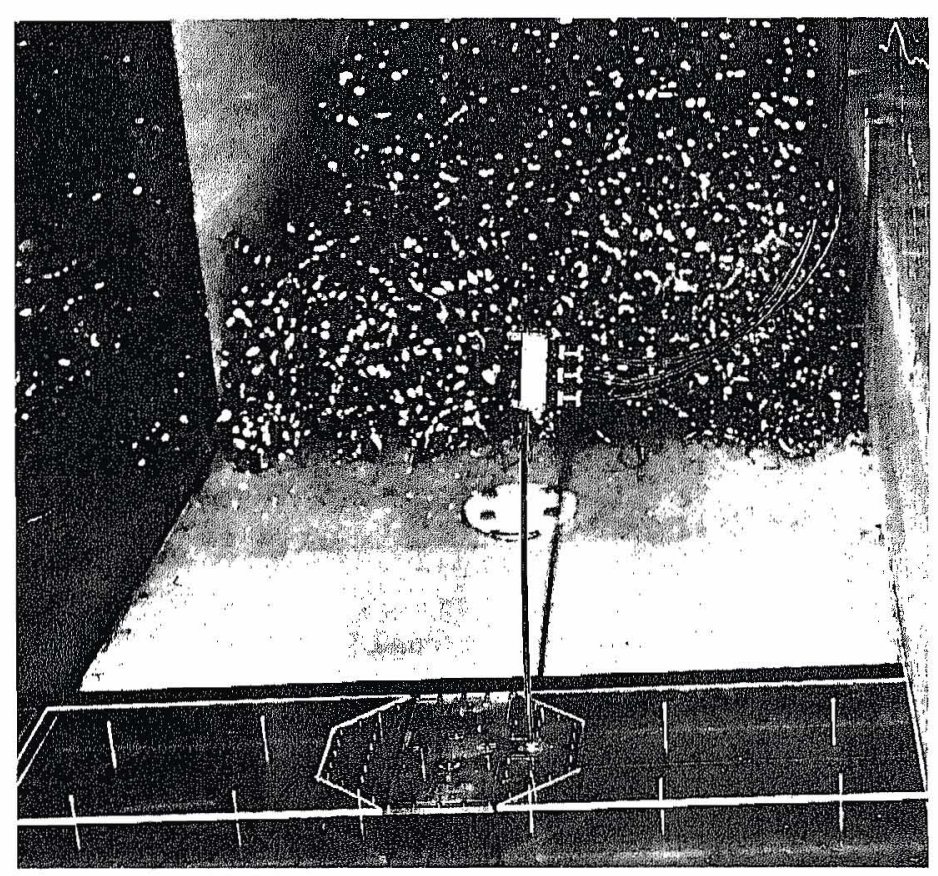

FIG. 2.-Flat Plate in Flume

the results a phase shift was made that alined the crest to crest experimental data with the $\sigma t=0$ base used in the analysis.

\section{Comparison of Experiment and Theory}

A first series of experiments was performed with $d=0.8 \mathrm{ft}(0.24 \mathrm{~m})$ and $s=0.6 \mathrm{ft}(0.18 \mathrm{~m})$ corresponding to $d^{\prime}=0.727$ and $s^{\prime}=0.545$, respectively. Figs. $4(a), 4(b)$, and $4(c)$ show results for frequencies of $0.92 \mathrm{~Hz}, 1.15 \mathrm{~Hz}$, and $1.38 \mathrm{~Hz}$ which correspond to $\lambda^{\prime}=4.29,3.17$, and 2.35 , respectively, the latter calculated with the aid of the intermediate water wave speed relation, $C=\sqrt{g / k \tanh k h}$. The corresponding Froude numbers, $F$, in the same sequence, are $0.8,1.0$, and 1.2. In Fig. 4 the data points represent pressure coefficient $\Delta p / \rho g a$ distributions for the wave positions shown in Fig. 5. 
Pressure coefficient distributions calculated from the theory are shown as solid curves on the same figures. The sets of integral equations (Eqs. 20 and 21) were replaced by 10 simultaneous linear equations that were solved numerically with the aid of a computer. In this case six reflections in both surface and bottom were considered, thus 13 terms of $G\left(\xi^{\prime}, x^{\prime}\right)$ were used.

In all cases, the general shape of the pressure distributions varies similarly with wave positions. When $\sigma t=0$, the crest has just passed over the plate and a node is over the leading edge. In this case $\Delta p$ is negative over the entire plate; the plate is being thrust down. When $\sigma t=\pi / 2$, the trough is over the

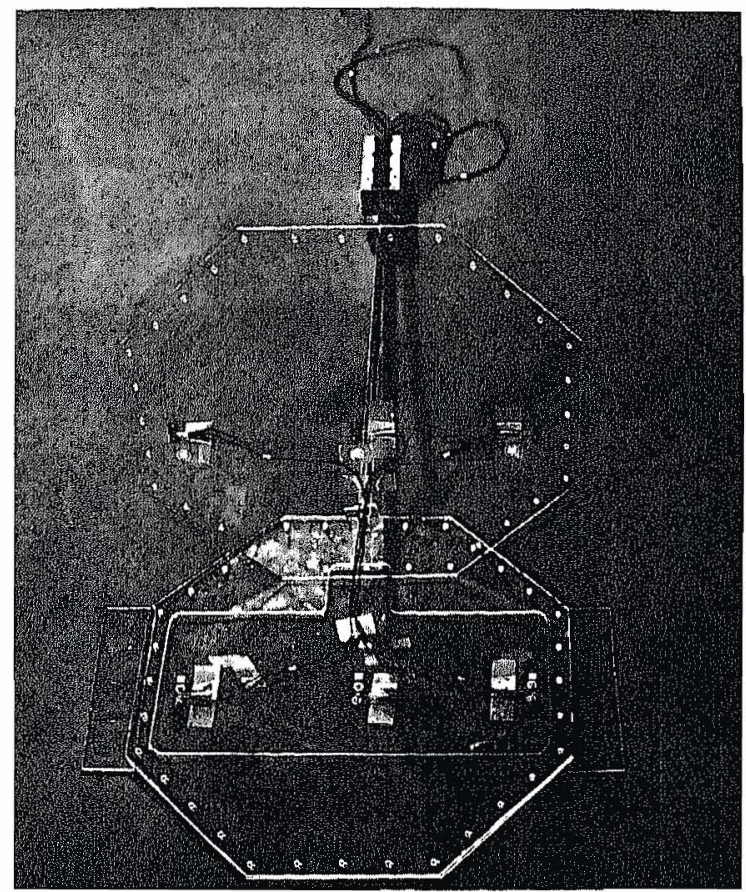

FIG. 3.-Construction of Plate

leading edge and $\Delta p$ is greater (positive) over the first portion of the plate and smaller or negative over the remainder. At $\sigma t=\pi$, the trough has passed over the plate and a node is over the leading edge. The plate is thrust upward as $\Delta p$ is positive over the entire plate. When $\sigma t=3 \pi / 2$, the crest is over the leading edge and $\Delta p$ is greater or negative over the first portion and smaller or positive over the remainder.

For the cases depicted in Figs. 4(a) and $4(b)$ the agreement between experiment and theory is quite good. These correspond to Froude numbers of 0.8 and 1.0, respectively. In Fig. 4(c), Froude number 1.2, the agreement is beginning to deteriorate although the general shape of the pressure distribution is correct. Thus, it is likely that the small Froude number approximation breaks down somewhere near unity.

A second series of experiments was conducted with $d=0.4 \mathrm{ft}(0.12 \mathrm{~m})$ and 


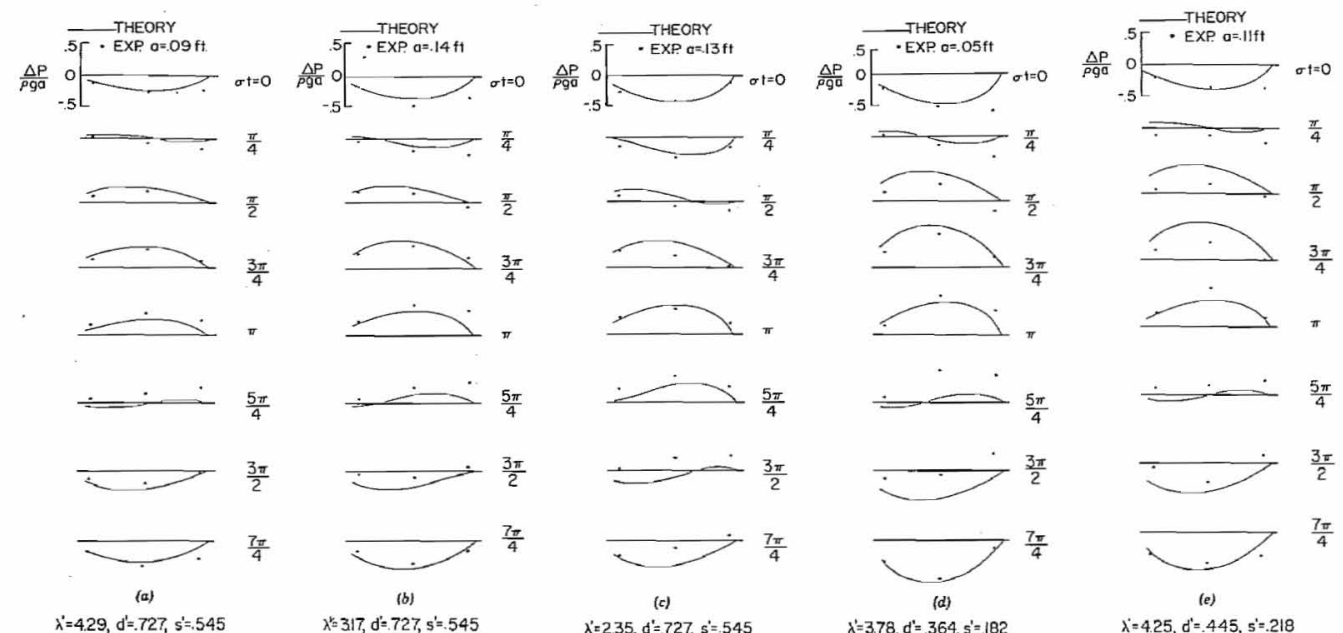

FIG. 4-Pressure Distributions (1 ft $=0.305 \mathrm{~m}$ ) 


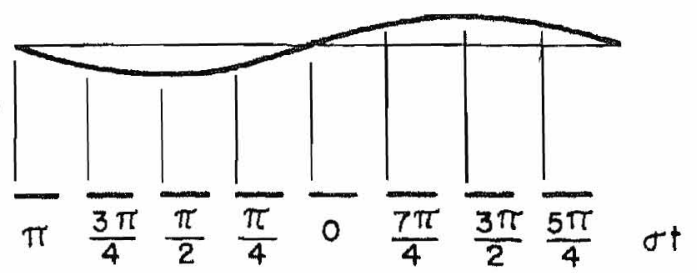

FIG. 5.-Positions of Leading Edge Relative to Wave

$s=0.2 \mathrm{ft}(0.06 \mathrm{~m})$ corresponding to $d^{\prime}=0.364$ and $s^{\prime}=0.182$, respectively. Fig. $4(d)$ shows the results for a frequency of $0.83 \mathrm{~Hz}$ corresponding to $\lambda^{\prime}=3.78$ and $F=0.4$. Again, the agreement between experiment and theory is good, except at $5 \pi / 4$ and $3 \pi / 2$. It should be pointed out that the waveforms were not as near sinusoidal in this case as the previous case. The surface profiles were somewhat triangular, undoubtedly contributing to the discrepancy.

Finally, a series of experiments were conducted using only the octagonally shaped central portion of the plate visible in Figs. 2 and 3. Fig. 4(e) shows results of a single test condition where $d=0.49 \mathrm{ft}(0.15 \mathrm{~m})$ and $s=0.25 \mathrm{ft}$ $(0.08 \mathrm{~m})$ corresponding to $d^{\prime}=0.44$ and $s^{\prime}=0.22$. The dimensionless wavelength was $\lambda^{\prime}=4.25$ and the Froude number, $F=0.3$. It can be seen from Fig. $4(e)$ that the agreement between experiment and theory is quite good qualitatively but that quantitatively some of the measured pressures are somewhat lower than predicted by the two-dimensional theory. This is to be expected, however, because of pressure venting around the edges of the plate. Also, the wave amplitude was quite large compared to the submergence, which results in asymmetry in the magnitude of the velocity distributions for wave positions one-half cycle apart.

Utilizing the hydrodynamic mass concept, the force coefficient can be defined as

$F=C^{\prime} M \frac{\partial v_{w}}{\partial t}=m_{h} \frac{\partial v_{w}}{\partial t} \ldots \ldots \ldots \ldots \ldots \ldots$

in which $C^{\prime}=$ force coefficient; $M=$ mass of the displaced fluid; and $m_{h}$ $=$ hydrodynamic mass.

The force per unit width can be obtained by integrating the pressure along the plate i.e.

$\frac{F}{L}=1 \int_{0}^{1} \Delta p d x^{\prime} \ldots \ldots \ldots \ldots \ldots \ldots \ldots \ldots \ldots \ldots$

By substitution of Eqs. 14 and 24 into Eq. 23, the force coefficient can be evaluated

$$
\begin{aligned}
& C^{\prime}=\frac{\lambda^{\prime}}{2 \pi} \frac{\cosh 2 \pi d^{\prime} / \lambda^{\prime}}{\sinh 2 \pi\left(d^{\prime}-s^{\prime}\right) / \lambda^{\prime}} \int_{0}^{1} \frac{\Delta P\left(x^{\prime}, t^{\prime}\right)}{\rho g a} d x^{\prime} . \\
& =-2 \pi \int_{0}^{1} \int_{0}^{x^{\prime}}\left[A\left(\xi^{\prime}\right) \cos t^{\prime}-B\left(\xi^{\prime}\right) \sin t^{\prime}\right] d \xi^{\prime} d x^{\prime} \ldots \ldots \ldots \ldots
\end{aligned}
$$


Using the maximum absolute value $C^{\prime} m=\left|C^{\prime}\right|_{\max } \ldots \ldots \ldots \ldots \ldots \ldots \ldots$

we can examine the dependence of the maximum force on various parameters.

In comparing Eq. 23 and the results of Meyerhoff (4), the hydrodynamic mass is

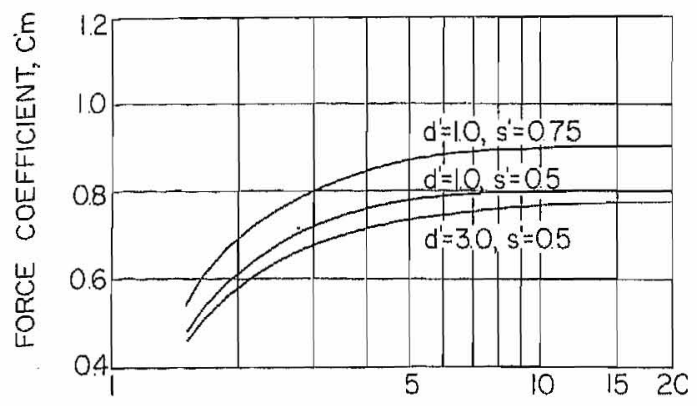

WAVE LENGTH / PLATE LENGTH, $\lambda^{\prime}$

FIG. 6.-Force Coefficient

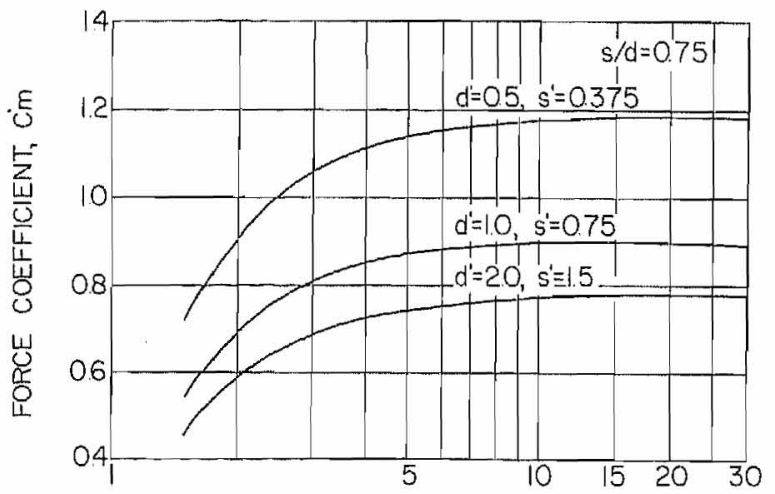

WAVE LENGTH/ PLATE LENGTH, $\lambda^{\prime}$

FIG. 7.-Force Coefficient, $S / D$ Fixed

$m_{h}=\frac{J \pi \rho}{4} l^{2} L \ldots \ldots \ldots \ldots \ldots \ldots \ldots \ldots \ldots \ldots \ldots \ldots \ldots \ldots \ldots \ldots$

Since the factor, $J \pi / 4$, corresponds to $C^{\prime} m$ and $J \rightarrow l$ as $L \rightarrow \infty$, we should expect $C^{\prime} m=0.78$ using this approach.

The force coefficient, $C^{\prime} m$, has been plotted with the wavelength to plate length ratio, $\lambda^{\prime}$, in Figs. 6 and 7 . Curves are plotted for various values of relative depth $d^{\prime}$ and submergence $s^{\prime}$. All curves show independence of $\lambda^{\prime}$ for large $\lambda^{\prime}$, reflecting a more uniform velocity distribution. At the greater 
relative depths, when the plate is not near the surface or bottom, it can be seen that $C^{\prime} m \rightarrow 0.78$ for large $\lambda^{\prime}$ as would be expected in the absence of boundary effects.

Fig. 6 shows that for fixed $\lambda^{\prime}$ and $d^{\prime}$ the force coefficient increases as the relative submergence is increased from 0.5 to 0.75 and that for fixed $\lambda^{\prime}$ and $s^{\prime}$ the coefficient increases as the relative depth is decreased from 3.0 to 1.0 . The curves in Fig. 7 have been computed holding $s / d=0.75$. Here, again, the force coefficient increases for decreasing relative depth for fixed $\lambda^{\prime}$.

\section{Conclusions}

The two-dimensional theory predicts the pressure distribution, induced by sinusoidal gravity waves, on a thin two-dimensional horizontal plate the width of which is not small compared to the wavelength. Because the theory is physically based on the concept of a vortex sheet and because of the subsequent approximate satisfaction of the free surface boundary condition, $F$ small, the mathematical complexity of the wave force problem is greatly reduced making application feasible. A comparison of the two-dimensional theory with measured pressures on an octagonal flat plate showed that the theory slightly overestimates the magnitude of the differential pressures.

The force coefficient, $C^{\prime} m$, becomes constant for large $\lambda^{\prime}$ at fixed $d^{\prime}, s^{\prime}$ and agrees with typical hydrodynamic masses provided surface and bottom effects are not too important.

Because the theory is linear, solutions are additive. More complex waveforms could, therefore, be accommodated by decomposing the waves into Fourier components. Further, the method could be applied to forms of finite thickness by the inclusion of suitable distributions of sources, sinks, and doublets.

\section{ACKNOWLEDGMENTS}

The basic research was conducted using the facilities of Alden Research Laboratories of Worcester Polytechnic Institute and was initiated under financial support from the American Electric Power Service Corporation.

\section{Appendix 1.-References}

1. Garrison, C. J., and Rao, V. S., "Interaction of Waves with Submerged Objects, Journal of the Waterways, Harbors and Coastal Engineering Division, ASCE, VC 94, No. WW2, Proc. Paper 8111, May, 1971, pp. 259-277.

2. Herbich, J. B., and Shank, G. E., "Forces Due to Waves on Submerged Structures," Journal of the Waterways, Harbors, and Coastal Engineering Division, ASCE, Vol. 97, No. WW1, Proc. Paper 7870, February, 1971, pp. 57-71.

3. Kuethe, A. M., and Schetzer, J. D., "The Thin Airfoil," Foundations of Aerodynamics, 2nd ed., John Wiley and Sons, Inc., New York, N.Y., 1963, pp. 75-93.

4. Meyerhoff, W. K., "Added Mass of Thin Rectangular Plates Calculated From Potential Theory," Journal of Ship Research, June, 1970, pp. 100-111.

5. Milne-Thomson, L. M., "Rectilinear Vortices," Theoretical Hydrodynamics, 4th ed., The MacMillian Company, New York, N.Y., 1960, pp. 372-373.

6. Morison, J. R., et al., "The Force Exerted by Surface Waves on Piles," Petroleum Transactions, American Institute Mining Engineers, Vol, 189, 1950, pp. 149-154. 
7. Robertson, J. M., "Acquisition of Potential Solutions," Hydrodynamics in Theory and Application, Prentice-Hall, Inc., Englewood Cliffs, N.J., 1965, pp. 251-252.

\section{Appendix II.-Notation}

The following symbols are used in this paper:

$A, B=$ functions defined by Eq. $19 ;$

$a=$ wave amplitude;

$C^{\prime}=$ force coefficient;

$C^{\prime} m=$ maximum absolute value of $C^{\prime}$;

$c=$ wave speed;

$d=$ fluid depth;

$d^{\prime}=d^{\prime} / l=$ dimensionless depth;

$\begin{aligned} F & =\text { force; } \\ F=\sqrt{s / g} & =\text { Froude number }\end{aligned}$

$G=$ function defined by Eq. 16;

$g=$ gravitational acceleration;

$J=$ added mass coefficient;

$k=2 \pi / \lambda=$ wave number;

$k^{\prime}=2 \pi / \lambda^{\prime}=$ dimensionless wave number;

$L=$ plate length;

$l=$ plate width;

$M=$ mass of displaced fluid;

$m_{h}=$ hydrodynamic mass;

$p=$ pressure;

$p^{+}=$pressure on upper surface;

$p^{-}=$pressure on lower surface;

$s=$ plate submergence;

$s^{\prime}=s / l=$ dimensionless submergence;

$t=$ time;

$t^{\prime}=\sigma t=$ dimensionless time;

$U=$ function defined in text;

$u=$ velocity in $x$ direction;

$u_{i}=$ velocity in $x$ direction due to perturbation;

$u_{w}=$ velocity in $x$ direction due to wave;

$v=$ velocity in $y$ direction;

$v_{i}=$ velocity in $y$ direction due to perturbation;

$v_{w}=$ velocity in $y$ direction due to wave;

$x=$ horizontal coordinate;

$x^{\prime}=x / l=$ dimensionless horizontal coordinate;

$y=$ vertical coordinate;

$\gamma=$ strength of vortex sheet;

$\Delta p=p^{-}-p^{+}=$differential pressure;

$\lambda=$ wavelength;

$\lambda^{\prime}=\lambda / l=$ dimensionless wavelength;

$\xi=$ dummy variable;

$\xi^{\prime}=\xi / l=$ dimensionless dummy variable;

$p=$ fluid density; 


$$
\begin{aligned}
\Sigma & =\text { represents additional images; } \\
\sigma=k c & =\text { angular frequency; } \\
\phi_{i} & =\text { perturbation potential; } \\
\phi_{w} & =\text { potential of incident wave; } \\
\phi_{1} & =\text { potential due to plate; } \\
\phi_{2} & =\text { potential of first image in bottom; and } \\
\phi_{3} & =\text { potential of first image in surface. }
\end{aligned}
$$

